\title{
PROCESSO DE TOMADA DE DECISÃO SOB INCERTEZA EM INVESTIMENTOS INTERNACIONAIS NA EXPLORAÇÃO \& PRODUÇÃO OFFSHORE DE PETRÓLEO: UMA ABORDAGEM MULTICRITÉRIO
}

\author{
Marcus Vinicius Margueron \\ Programa de Planejamento Energético (PPE) / COPPE \\ Universidade Federal do Rio de Janeiro (UFRJ) \\ marguero@,finep.gov.br \\ Lucio Guido Tapia Carpio * \\ Programa de Planejamento Energético (PPE) / COPPE \\ Universidade Federal do Rio de Janeiro (UFRJ) \\ guido@ppe.ufrj.br \\ * Corresponding author / autor para quem as correspondências devem ser encaminhadas \\ Recebido em 02/2004; aceito em 06/2005 após 1 revisão \\ Received February 2004; accepted June 2005 after one revision
}

\begin{abstract}
Resumo
Este trabalho apresenta o desenvolvimento teórico sobre a análise de projetos de exploração e produção de petróleo, desde a avaliação econômica tradicional através do Fluxo de Caixa Descontado até a apresentação da Teoria da Utilidade Multiatributo (MAUT), que possibilita a consideração dos riscos e incertezas típicos dos investimentos internacionais no setor upstream do petróleo em um novo ambiente competitivo entre as firmas. Por fim, o trabalho inclui um estudo de caso sobre a aplicação da MAUT na priorização de dez oportunidades exploratórias offshore ao redor do mundo oferecidas a uma multinacional brasileira fictícia de acordo com as preferências dos tomadores de decisão sobre aspectos operacionais, políticos e tecnológicos.
\end{abstract}

Palavras-chave: exploração \& produção de petróleo; teoria da utilidade multiatributo; investimentos sob incerteza.

\begin{abstract}
This paper presents the theoretical developments in techniques of decision-making analysis in petroleum exploration and production projects, from economic evaluation using Discount Cash Flow (DCF) up to and including a presentation of the Multi-Attribute Utility Theory (MAUT), which enables consideration of typical risks and uncertainties in international petroleum upstream investments in a new competitive situation among firms. Finally, a case study of the MAUT was developed and applied to define priorities among ten offshore exploration opportunities around the world offered to a fictitious Brazilian petroleum multinational company, according to decision-makers' preferences regarding operational, political and technological issues.
\end{abstract}

Keywords: petroleum exploration \& production; multiattribute utility theory; investments under uncertainties. 


\section{Introdução}

Tendo em vista que na última década as empresas de petróleo aumentaram substancialmente o patamar de seus investimentos em atividades de exploração e produção (E\&P) no exterior, focaremos esse artigo exatamente no cenário internacional, cada vez mais globalizado, onde a competitividade e também as possibilidades de parcerias incentivam as empresas a adotarem técnicas quantitativas mais avançadas de avaliação de projetos de exploração e produção de petróleo para reduzirem os riscos e incertezas nos investimentos.

Os tradicionais métodos de análise econômico-financeira se baseiam em considerações sobre o Fluxo de Caixa Descontado (FCD). Para a análise econômica de projetos que apresentam somente variáveis determinísticas, as metodologias, como a do Valor Presente Líquido (VPL), são extremamente recomendadas. No entanto, em análises de investimentos onde estejam envolvidos eventos estocásticos, apresentando incertezas relevantes a respeito dos resultados futuros, esses métodos não devem ser aplicados isoladamente. Eles precisam estar amparados por ferramentas mais refinadas de análise de decisão, como a Teoria da Decisão.

Apesar de os conceitos básicos da Teoria da Decisão já terem sido formulados há mais de 300 anos, apenas na década de 70 que Newendorp (1975) passou a aplicá-los diretamente na análise de investimentos na exploração e produção de petróleo.

O critério utilizado para selecionar a melhor estratégia em projetos que apresentem incertezas deixa então de ser a maximização do VPL, passando a ser aquele que maximiza o Valor Monetário Esperado (VME), que é dado pela seguinte formulação:

$$
V M E=p_{s} \times V P L_{s}+p_{f} \times V P L_{f}
$$

$\mathrm{VPL}_{\mathrm{s}} \Rightarrow$ Valor Presente Líquido do Sucesso;

$\mathrm{VPL}_{\mathrm{f}} \Rightarrow$ Valor Presente Líquido do Fracasso; e $\mathrm{p}_{\mathrm{s}} \Rightarrow$ Probabilidade de Sucesso;

$\mathrm{p}_{\mathrm{f}} \Rightarrow$ Probabilidade de Fracasso.

A grande limitação da metodologia do VME está na desconsideração do grau de exposição da organização a perdas. Dessa forma, é necessário que façamos uso de uma ferramenta analítica capaz de manter o determinismo matemático do critério de maximização do VME, mas também de considerar o posicionamento dos tomadores de decisão frente ao risco financeiro. A ferramenta a que nos referimos é a Teoria da Utilidade.

\section{Teoria da Utilidade}

As publicações de Newendorp (1975), Walls (1995) e Nepomuceno (1997) mostram que a indústria do petróleo já vem utilizando essas técnicas de risco na avaliação de projetos de exploração e produção. Por trás da aplicação desta Teoria encontra-se a análise do complexo comportamento dos seres humanos frente ao risco, podendo ser avesso, indiferente ou propenso.

Tradicionalmente, as companhias de petróleo, apesar de apresentarem grande capacidade financeira de suportar perdas, possuem capital exploratório inferior ao seu estoque de prospectos minerais, fazendo com que apresentem um certo nível de aversão ao risco devido à limitação orçamentária.

A melhor decisão a ser tomada em investimentos sob risco e incerteza passa a ser definida com auxílio da função-utilidade através do critério de maximização do Valor Esperado da 
Utilidade (VEU), analogamente ao que ocorria com a metodologia de maximização do Valor Monetário Esperado (VME) da base da Teoria da Decisão.

O Valor Esperado da Utilidade de um projeto é dado por:

$$
V E U=p_{s} \times U\left(V P L_{s}\right)+p_{f} \times U\left(V P L_{f}\right)
$$

Para determinado indivíduo ou organização existe a mesma preferência ou utilidade entre o recebimento da quantia do Equivalente Certo $(\mathrm{EqC})$ e a participação no evento incerto e arriscado.

$$
U(E q C)=V E U
$$

Observamos, pela definição anterior, que podemos maximizar o VEU através da maximização da utilidade do $\mathrm{EqC}$, ou simplesmente pela própria maximização dele.

A Teoria da Utilidade Multiatributo (MAUT) nada mais é que a Teoria da Utilidade aplicada a processos decisórios que consideram múltiplos critérios, muitas vezes conflituosos. Ela define uma função-utilidade multiatributo composta por funções-utilidade individuais.

$$
U(y, z)=f\left\{U_{y}(y), U_{z}(z)\right\}
$$

Essa possibilidade de definir as funções-utilidade de cada um dos atributos e depois uni-las em uma única função multicritério é um grande facilitador na modelagem. No entanto, precisamos seguir corretamente os axiomas teóricos no processo de consolidação das funções individuais em uma única função-utilidade combinada (Gomes et al., 2002).

\section{Seleção dos Atributos}

A decisão de aplicar o capital de uma multinacional brasileira em prospectos internacionais offshore de petróleo é realizada através de uma análise conjunta das condições oferecidas no momento da contratação do projeto, mas, principalmente, das perspectivas futuras, quando deve ser realizada a importante análise dos riscos financeiros.

Entretanto, o risco de perdas financeiras ao se decidir por um investimento internacional em $\mathrm{E} \& \mathrm{P}$ offshore é influenciado por variáveis operacionais, políticas e tecnológicas. Todas elas são consideradas mais ou menos relevantes de acordo com o comportamento do empresário fator extremamente subjetivo que consegue ser capturado pela MAUT.

\subsection{Risco operacional}

O risco financeiro associado às atividades de exploração e produção de petróleo está intimamente ligado às consideráveis possibilidades de perdas relacionadas aos investimentos no sentido de descobrir novas reservas de óleo.

No setor de exploração e produção de petróleo a maioria das empresas apresenta certa dificuldade na determinação exata de sua função-utilidade, e, de acordo com Cozzolino (1977), a maioria delas desconhece sua função. Estudando o processo de tomada de decisão dos gerentes das principais companhias multinacionais de petróleo, Walls (1995) chegou à seguinte função-utilidade exponencial para essas empresas: 


$$
U(x)=-e^{-c x}
$$

$\mathrm{x} \Rightarrow$ valor monetário; $\quad \mathrm{e} \quad \mathrm{c} \Rightarrow$ coeficiente de aversão ao risco operacional.

Aplicando a função-utilidade (5) à definição de que a utilidade do Equivalente Certo corresponde ao Valor Esperado da Utilidade (VEU), conforme mostra a formulação (3), temos o Equivalente Certo dos projetos no setor upstream, cuja demonstração matemática pode ser encontrada no Anexo 1, já considerando o nível ótimo de participação (np).

$$
E q C(n p)=-\frac{1}{c} \ln \left(p e^{-c\left(V P L_{1}\right) n p}+(1-p) e^{-c\left(V P L_{2}\right) n p}\right)
$$

\subsection{Risco político}

A globalização da economia resultou em uma maior preocupação com a discussão do risco político. Com a integração internacional cada vez maior das atividades produtivas e das estratégias empresariais, a maior dedicação à compreensão da dinâmica das questões políticas, econômicas e sociais nacionais e a consideração desses processos nas análises dos investidores globais são cada vez mais imprescindíveis para o sucesso e prosperidade das companhias que se lançam em negócios internacionais, uma vez que interferências de ordem política podem resultar em grandes perdas financeiras.

Estabilidade, clareza e credibilidade na relação contratual e regulatória são fundamentais para a atratividade de investimentos estrangeiros, principalmente pelo fato de as aplicações em atividades de Exploração e Produção (E\&P) serem vultuosas e de longo período de maturação.

A análise de risco político de nosso estudo de caso utilizará as informações mais atualizadas a respeito das realidades de cada um dos países onde estejam sendo oferecidas oportunidades exploratórias. Procuraremos encarar o risco político com um enfoque bastante integrado e multidimensional, considerando a ponderação das seguintes macrovariáveis:

- Avaliação por informações e indicadores, que considera aspectos políticos, econômicos, sociais e comercial/mercadológicos das nações a partir de pesquisas de alguns especialistas em risco político e informações atualizadas da publicação oficial da CIA em 2002 intitulada “The World Factbook 2002”. The World Factbook (2002);

- Resultados da avaliação de Hargreaves et al. (1994) - especialistas em risco político no setor mineral;

- Classificação dos países quanto à percepção de corrupção pela Transparência Internacional em 2002. Folha de São Paulo (2002); e

- Classificação de risco de crédito dos países por uma agência internacional de "rating". Global Invest (2003).

\subsection{Risco tecnológico}

Alguns prospectos exigem grandes inovações tecnológicas para tornar sua produção viável. No entanto, muitas empresas decidem enfrentar o ônus financeiro de um enorme desafio tecnológico, ou seja, o risco tecnológico, motivadas por um objetivo maior de diferenciação 
competitiva ao dominar uma tecnologia inovadora e exclusiva de produção em águas profundas, que poderá viabilizar economicamente outras oportunidades exploratórias muito mais rentáveis, antes inviáveis.

Devemos deixar de lado a mentalidade estreita de que as iniciativas de desenvolvimentos tecnológicos são simplesmente despesas ou gastos, e passar a visualizá-las como investimentos. Atualmente verificamos parcerias entre diversos centros de pesquisa e o CENPES (Centro de Pesquisas da Petrobras) numa busca constante de incremento de rentabilidade decorrente de saltos tecnológicos pretendidos com inovações para produção em águas profundas, financiadas com recursos próprios da Petrobras ou do governo através do CTPETRO (Fundo Setorial do Petróleo, Gás e Petroquímica), operacionalizado pela Financiadora de Estudos e Projetos - FINEP.

Apesar de se saber que as reservas de petróleo mundiais restantes se encontrarão cada vez mais em locais de difícil acesso, a maiores profundidades, grande parte dos empresários do setor ainda não considera a influência da componente tecnológica ao tomar decisões de alocação de recursos, em razão de os benefícios decorrentes do desenvolvimento tecnológico serem de difícil mensuração.

Para tentar resolver esse problema, Suslick et al. (2001) propuseram um inédito modelo de otimização de alocação de recursos para empresas petrolíferas de E\&P, considerando a profundidade e a lâmina d'água sob a qual se encontra o possível reservatório bons indicadores para a avaliação do volume de desenvolvimentos tecnológicos possíveis de serem alcançados. Os ganhos financeiros relativos a essas variáveis tecnológicas estão associados à redução de despesas de investimento, de custos operacionais, ao aumento de produção de petróleo e de reservas provadas.

Eles assumem que uma curva $\mathrm{S}$ representa graficamente a função-utilidade da variável tecnológica para os empresários do setor petrolífero dispostos a considerar a utilidade dos avanços tecnológicos em seus processos decisórios.

Então Suslick et al. (2001) chegaram à formulação para a função-utilidade tecnológica $\mathrm{U}_{\text {tec }}$, que concede igual ponderação para a utilidade da lâmina d'água (ld) e para a profundidade do reservatório (pr).

$$
\begin{gathered}
U_{t e c}(l d, p r)=\frac{U_{t e c}(l d)+U_{t e c}(p r)}{2} \\
U_{t e c}(l d, p r)=\frac{\frac{1}{\left(1+76,5 e^{-0,005(l d)}\right)}+\frac{1}{\left(1+600 e^{-0,002(p r)}\right)}}{2}
\end{gathered}
$$

\section{Estudo de Caso}

A MAUT será aplicada na priorização de projetos internacionais de exploração e produção offshore de petróleo de uma companhia multinacional brasileira fictícia. Será determinado o nível ótimo de participação em cada uma das oportunidades exploratórias, inicialmente com a análise exclusiva do risco operacional, e, sucessivamente, introduzindo de forma cumulativa a avaliação dos riscos políticos e tecnológicos. Buscaremos a otimização do sistema composto pelos seguintes objetivos: 
- Maximizar retornos financeiros decorrentes de características operacionais, representadas fundamentalmente pelo Valor Presente Líquido (VPL) e pela probabilidade de sucesso exploratório (p);

- Minimizar perdas financeiras relativas aos aspectos políticos; e

- Maximizar possibilidade de avanços tecnológicos em produção de petróleo em águas profundas.

Foi inevitável a consideração das informações operacionais disponíveis da Petrobras Internacional Braspetro para a definição da estratégia empresarial de internacionalização, do porte e do volume de reservas de nossa corporação fictícia e também dos países onde existem as oportunidades de negócios.

Todo o processo de modelagem da MAUT deve estar suportado por uma rígida estrutura axiomática, sendo este processo denominado de elicitação. Este trabalho em momento algum buscou simplificações que pudessem comprometer a qualidade da modelagem das funçõesutilidades combinadas apresentadas, mas, pelo contrário, seguiu todas as recomendações para a aplicação da MAUT como Teoria. A importância de se utilizar a Teoria da Utilidade Multiatributo como Teoria e não como Método está muito bem detalhada em Gomes et al. (2002). Verificamos que os tomadores de decisão consideraram os atributos independentes em utilidade e também aditivamente independentes - principais condicionantes da MAUT para aplicar com segurança a forma aditiva da função-utilidade multiatributo, na qual inexistem interações entre os atributos.

\subsection{Risco operacional}

Tabela 1 - Informações operacionais e tecnológicas.

\begin{tabular}{|c|c|c|c|c|c|}
\hline $\begin{array}{c}\text { Localização da } \\
\text { Bacia }\end{array}$ & $\begin{array}{c}\text { Valor Presente } \\
\text { Líquido (VPL) } \\
\text { (milhões de US\$) }\end{array}$ & $\begin{array}{c}\text { Custos de aquisição } \\
\text { e exploração (C) } \\
\text { (milhões de US\$) }\end{array}$ & $\begin{array}{c}\text { Probabilidade de } \\
\text { Sucesso (p) } \\
(\%)\end{array}$ & $\begin{array}{c}\text { Lâmina d'água } \\
\text { (metros) }\end{array}$ & $\begin{array}{c}\text { Profundidade do } \\
\text { Reservatório } \\
\text { (metros) }\end{array}$ \\
\hline Angola & 1000 & 100 & 55 & 2000 & 4000 \\
\hline Argentina & 280 & 25 & 30 & 100 & 3000 \\
\hline Canadá & 400 & 30 & 18 & 1000 & 3500 \\
\hline Casaquistão & 350 & 40 & 20 & 400 & 2500 \\
\hline China & 500 & 50 & 22 & 500 & 3400 \\
\hline EUA & 950 & 60 & 14 & 2500 & 6000 \\
\hline Nigéria & 900 & 85 & 45 & 1750 & 3050 \\
\hline Reino Unido & 450 & 15 & 12 & 1200 & 4100 \\
\hline Rússia & 700 & 55 & 21 & 800 & 3300 \\
\hline Venezuela & 850 & 40 & 35 & 2200 & 5900 \\
\hline
\end{tabular}

Durante a década de 80, Walls et al. (1996) realizaram um estudo empírico nas 18 maiores companhias de petróleo e verificaram que, de um modo geral, elas apresentam tolerância ao risco operacional (T) igual a um quarto do seu capital exploratório anual (K), expresso em milhões de dólares. Como o capital exploratório anual da companhia fictícia é de US\$400 milhões, seu coeficiente de aversão ao risco operacional (c) é igual a: 


$$
c=\frac{1}{T}=\frac{4}{K}=\frac{4}{400}=0,01
$$

Partiremos agora para o cálculo do Equivalente Certo e do nível ótimo de participação em cada um dos projetos nacionais, como demonstraremos a seguir para o projeto angolano.

Aplicando a fórmula (9) aos níveis de participação em um intervalo de $10 \%$, construímos o gráfico da Figura 1, permitindo-nos definir visualmente o nível de participação (np) que maximiza o Equivalente Certo do projeto.

$$
E q C(n p)=-\frac{1}{c} \ln \left(p e^{-c(V P L) n p}+(1-p) e^{-c(-C) n p}\right)
$$

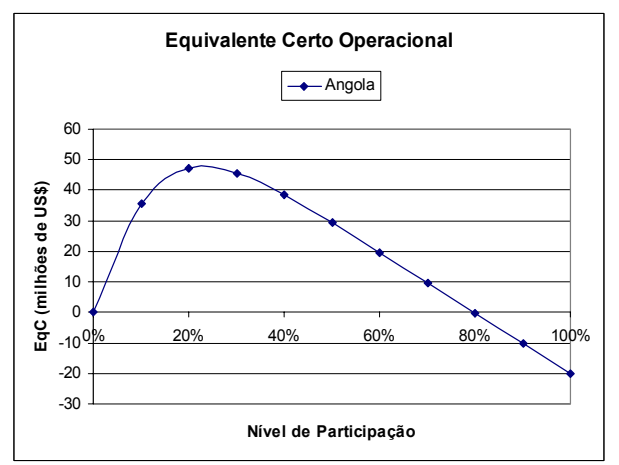

Figura 1 - Gráfico do Equivalente Certo $(\mathrm{EqC})$ do projeto angolano.

É importante mencionar que durante um leilão de oportunidades exploratórias é pouco comum alcançar o nível ótimo de participação (np) definido pelo modelo para maximizar o retorno financeiro de determinado bloco; afinal de contas, existem outras companhias concorrentes defendendo seus interesses particulares. Apesar disso, estamos considerando um cenário em que a empresa fictícia consiga adquirir as participações ótimas desejadas em cada uma das oportunidades exploratórias.

Ressaltamos que todas as utilidades apresentadas nesse trabalho foram normalizadas em uma escala de 0 a 1.

O Valor Esperado da Utilidade operacional $\left(\mathrm{VEU}_{\mathrm{op}}\right)$ de cada um dos projetos foi obtido a partir do Equivalente Certo através da formulação (10), demonstrada matematicamente no Anexo 2.

$$
V E U_{o p}=U_{o p}(E q C)=\frac{e^{-c(-100)}-e^{-c(E q C)}}{e^{-c(-100)}-e^{-c(1000)}}
$$

Outra forma de se chegar ao VEU de cada um dos projetos, só que de forma direta sem apresentar o Equivalente Certo de cada um deles, é através da equação (11), que já considera na primeira parcela, a probabilidade de sucesso exploratório (p) e o retorno (VPL) a ela associado e, em uma segunda parcela, a chance de fracasso do projeto (1-p), com o custo (-C) incorrido. 


$$
V E U_{o p}=p \times\left[\frac{e^{-c(-100)}-e^{-c(V P L)}}{e^{-c(-100)}-e^{-c(1000)}}\right]+(1-p) \times\left[\frac{e^{-c(-100)}-e^{-c(-C)}}{e^{-c(-100)}-e^{-c(1000)}}\right]
$$

Definimos o nível ótimo de participação, ou seja, aquele que otimiza o $\mathrm{VEU}_{\text {op }}$ dos tomadores de decisão, e chegamos aos seguintes resultados, apresentados agregados nos dois gráficos que seguem.
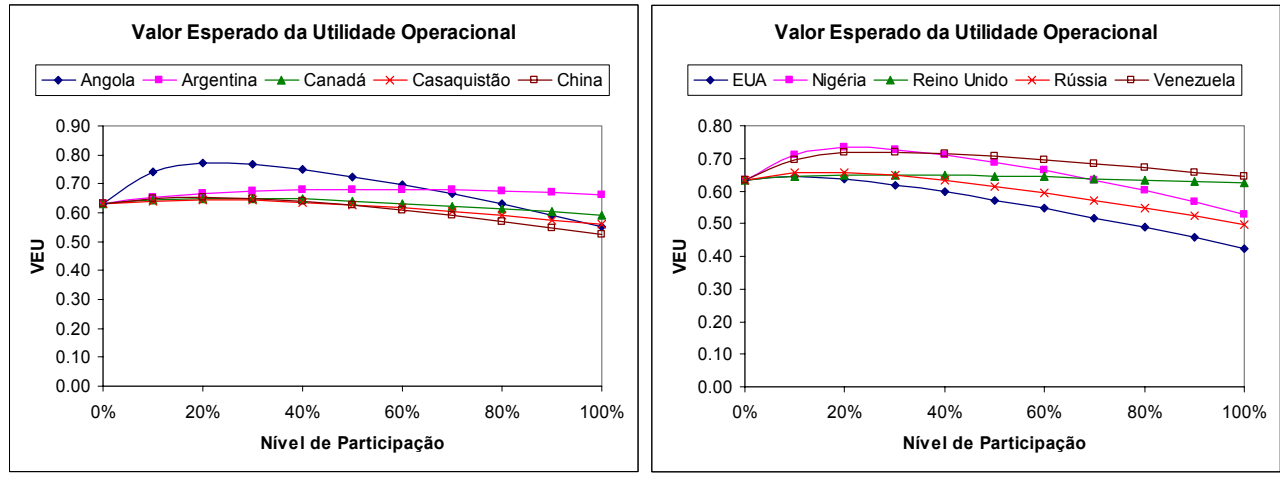

Figura 2 - Gráficos dos Valores Esperados das Utilidades operacionais $\left(\mathrm{VEU}_{\mathrm{op}}\right)$.

Tabela 2 - Classificação dos projetos nacionais considerando apenas o risco operacional.

\begin{tabular}{|c|c|c|c|}
\hline Países & VEU $_{\text {op }}$ & Participação Ótima & Classificação \\
\hline Angola & 0,7704 & $20 \%$ & $1^{\circ}$ \\
\hline Argentina & 0,6810 & $50 \%$ & $4^{\circ}$ \\
\hline Canadá & 0,6500 & $30 \%$ & $7^{\circ}$ \\
\hline Casaquistão & 0,6447 & $20 \%$ & $9^{\circ}$ \\
\hline China & 0,6531 & $20 \%$ & $6^{\circ}$ \\
\hline EUA & 0,6442 & $10 \%$ & $10^{\circ}$ \\
\hline Nigéria & 0,7328 & $20 \%$ & $2^{\circ}$ \\
\hline Reino Unido & 0,6499 & $30 \%$ & $8^{\circ}$ \\
\hline Rússia & 0,6565 & $20 \%$ & $5^{\circ}$ \\
\hline Venezuela & 0,7203 & $30 \%$ & $3^{\circ}$ \\
\hline
\end{tabular}

\subsection{Risco político}

Os resultados de nossa avaliação de risco político por informações e indicadores, que estão na Tabela 3 apresentados em uma escala de 0 a 100 - onde 0 representa risco mínimo e 100 risco máximo -, foram divididos em quatro aspectos: político, econômico, comercial/ mercadológico e social, considerados pelos tomadores de decisão com igual importância em cada um dos países. 
Tabela 3 - Avaliação por informações e indicadores.

\begin{tabular}{|c|c|c|c|c|c|}
\hline Países & $\begin{array}{c}\text { Aspecto } \\
\text { Político }\end{array}$ & $\begin{array}{c}\text { Aspecto } \\
\text { Econômico }\end{array}$ & $\begin{array}{c}\text { Aspecto } \\
\text { Comercial/ } \\
\text { Mercadológico }\end{array}$ & Aspecto Social & $\begin{array}{c}\text { Risco Político Nacional } \\
\text { por Informações e } \\
\text { Indicadores }\end{array}$ \\
\hline Angola & 95,00 & 95,00 & 80,00 & 95,00 & 91,25 \\
\hline Argentina & 80,00 & 85,00 & 50,00 & 65,00 & 70,00 \\
\hline Canadá & 35,00 & 30,00 & 35,00 & 25,00 & 31,25 \\
\hline Casaquistão & 85,00 & 65,00 & 85,00 & 70,00 & 76,25 \\
\hline China & 80,00 & 50,00 & 75,00 & 60,00 & 66,25 \\
\hline EUA & 40,00 & 25,00 & 30,00 & 45,00 & 35,00 \\
\hline Nigéria & 90,00 & 90,00 & 85,00 & 95,00 & 90,00 \\
\hline Reino Unido & 35,00 & 30,00 & 35,00 & 30,00 & 32,50 \\
\hline Rússia & 75,00 & 70,00 & 85,00 & 70,00 & 75,00 \\
\hline Venezuela & 85,00 & 75,00 & 55,00 & 75,00 & 72,50 \\
\hline
\end{tabular}

Apresentamos então a Tabela 4, que consolida os resultados obtidos para cada um dos cinco fatores analisados, com adequadas ponderações, e também explicita o risco político final de cada um dos projetos nacionais.

Tabela 4 - Resultados finais da metodologia própria de avaliação de risco político.

\begin{tabular}{|c|c|c|c|c|c|c|}
\hline $\begin{array}{c}\text { Localização da } \\
\text { Bacia }\end{array}$ & $\begin{array}{c}\text { Avaliação por } \\
\text { Informações e } \\
\text { Indicadores } \\
(20 \% \text { a 30\%) }\end{array}$ & $\begin{array}{c}\text { Percepção da } \\
\text { Realidade } \\
\text { Local } \\
(20 \% \text { a 30\%) }\end{array}$ & $\begin{array}{c}\text { Especialistas } \\
\text { em Risco } \\
\text { Político } \\
(30 \%)\end{array}$ & $\begin{array}{c}\text { Percepção da } \\
\text { Corrupção } \\
(10 \%)\end{array}$ & $\begin{array}{c}\text { Agência de } \\
\text { "Rating”" } \\
(10 \%)\end{array}$ & $\begin{array}{c}\text { Risco Político } \\
\text { Final } \\
(100 \%)\end{array}$ \\
\hline Angola & 91,25 & 85,00 & 100,00 & 83,00 & - & 91,56 \\
\hline Argentina & 70,00 & 70,00 & 41,67 & 72,00 & 75,00 & 62,20 \\
\hline Canadá & 31,25 & 30,00 & 25,00 & 10,00 & 00,00 & 23,84 \\
\hline Casaquistão & 76,25 & 70,00 & 75,00 & 77,00 & 35,00 & 70,04 \\
\hline China & 66,25 & 60,00 & - & 65,00 & 25,00 & 57,63 \\
\hline EUA & 35,00 & 40,00 & 33,33 & 23,00 & 00,00 & 30,80 \\
\hline Nigéria & 90,00 & 90,00 & 83,33 & 84,00 & - & 87,11 \\
\hline Reino Unido & 32,50 & 35,00 & 25,00 & 13,00 & 00,00 & 25,55 \\
\hline Rússia & 75,00 & 65,00 & 75,00 & 73,00 & 40,00 & 68,56 \\
\hline Venezuela & 72,50 & 75,00 & 66,67 & 75,00 & 59,00 & 70,27 \\
\hline
\end{tabular}

Quanto maior o grau de controle sobre a mídia e menor a experiência operacional da empresa ou de outras multinacionais do petróleo na região, maior foi importância e peso dados à percepção da realidade local pelos executivos da companhia e menor às avaliações por informações e indicadores.

Consideramos que a função-utilidade da companhia é a mais freqüente entre os tomadores de decisão do setor petrolífero mundial, e, além disso, que o coeficiente de aversão ao risco político é duas vezes superior ao operacional. Isso se deve ao fato de que, para a empresa fictícia, uma situação de intervenção do governo local sobre sua atividade produtiva é duas 
vezes mais temida que uma outra de fracasso exploratório motivado por variáveis operacionais.

Apresentamos a utilidade do risco político, cuja obtenção pode ser vista em Margueron (2003) (ver Anexo 3)

$$
U(R P)=\frac{e^{-2 c(91,56)}-e^{-2 c(R P)}}{e^{-2 c(91,56)}-e^{-2 c(23,84)}}
$$

Tabela 5 - Risco político e sua utilidade para os tomadores de decisão.

\begin{tabular}{|c|c|c|}
\hline Países & Risco Político & $\mathrm{U}(\mathrm{RP})$ \\
\hline Angola & 91,56000 & 0,00000 \\
\hline Argentina & 62,20000 & 0,27795 \\
\hline Canadá & 23,84000 & 1,00000 \\
\hline Casaquistão & 70,04075 & 0,18711 \\
\hline China & 57,62964 & 0,33785 \\
\hline EUA & 30,80000 & 0,82485 \\
\hline Nigéria & 87,11111 & 0,03237 \\
\hline Reino Unido & 25,55000 & 0,95468 \\
\hline Rússia & 68,56220 & 0,20317 \\
\hline Venezuela & 70,26605 & 0,18471 \\
\hline
\end{tabular}

Aplicaremos uma modelagem multiatributo que possibilitará a integração das percepções individuais de cada atributo em um único equacionamento matemático. Antes disso, é necessário que os tomadores de decisão explicitem a importância relativa dos dois atributos considerados até o momento, ou seja, o risco operacional e o risco político.

A equação (13) considera as variáveis operacionais, por meio da utilidade do Valor Presente Líquido (VPL) do investimento, obtida de maneira análoga à utilidade do EqC representada pela formulação (10), e políticas, através do resultado da utilidade dos riscos políticos (RP) nacionais.

$$
U_{o p, p o l}(V P L, R P)=k_{o p} \times U_{o p}(V P L)+k_{p o l} \times U_{p o l}(R P)
$$

$\mathrm{Na}$ definição das duas constantes de escala $\mathrm{k}_{\mathrm{op}}$ e $\mathrm{k}_{\mathrm{pol}}$, que assumem valores de 0 a 1 e somadas são iguais à unidade, devemos buscar o mesmo número de situações de indiferença para os tomadores de decisão, ou seja, que fiquem igualmente satisfeitos entre duas oportunidades de negócio apresentadas. $\mathrm{O}$ resultado foi que eles se consideraram igualmente preferíveis entre o maior VPL do projeto no Casaquistão e um menor risco político na Argentina para sua empresa multinacional. Sendo assim, pudemos definir que:

$$
\begin{aligned}
& U_{o p, p o l}\left(V P L_{C a s}, R P_{C a s}\right)=U_{o p, p o l}\left(V P L_{A r g}, R P_{A r g}\right) \\
& U_{o p, p o l}(350 ; 70,04)=U_{o p, p o l}(280 ; 62,20)
\end{aligned}
$$

Chegamos a um sistema com duas equações e duas incógnitas: 


$$
\begin{gathered}
\mid \begin{array}{l}
\left.k_{o p} \times U_{o p}(350)\right]+\left[k_{p o l} \times U_{p o l}(70,04)\right] \\
k_{o p}+k_{p o l}=1
\end{array}=\left[k_{o p} \times U_{o p}(280)\right]+\left[k_{p o l} \times U_{p o l}(62,20)\right] \\
k_{o p}=0,88965 \\
k_{p o l}=0,11035
\end{gathered}
$$

A obtenção do $\mathrm{VEU}_{\text {op,pol }}$ de cada um dos projetos, considerando todos os riscos operacionais e políticos, é possível através da aplicação da equação (14).

$$
\begin{aligned}
V E U_{o p, p o l} & =k_{o p}\left\{p \times\left[\frac{e^{-c(-100)}-e^{-c(V P L)}}{e^{-c(-100)}-e^{-c(1000)}}\right]+(1-p) \times\left[\frac{e^{-c(-100)}-e^{-c(C)}}{e^{-c(-100)}-e^{-c(1000)}}\right]\right\}+ \\
& +k_{p o l} \times\left[\frac{e^{-2 c(91,56)}-e^{-2 c(R P)}}{e^{-2 c(91,56)}-e^{-2 c(23,84)}}\right]
\end{aligned}
$$

Então otimizamos o valor esperado global das utilidades operacional e política para os tomadores de decisão, definindo o nível ótimo de participação em cada um dos projetos, conforme mostram os dois gráficos que seguem.
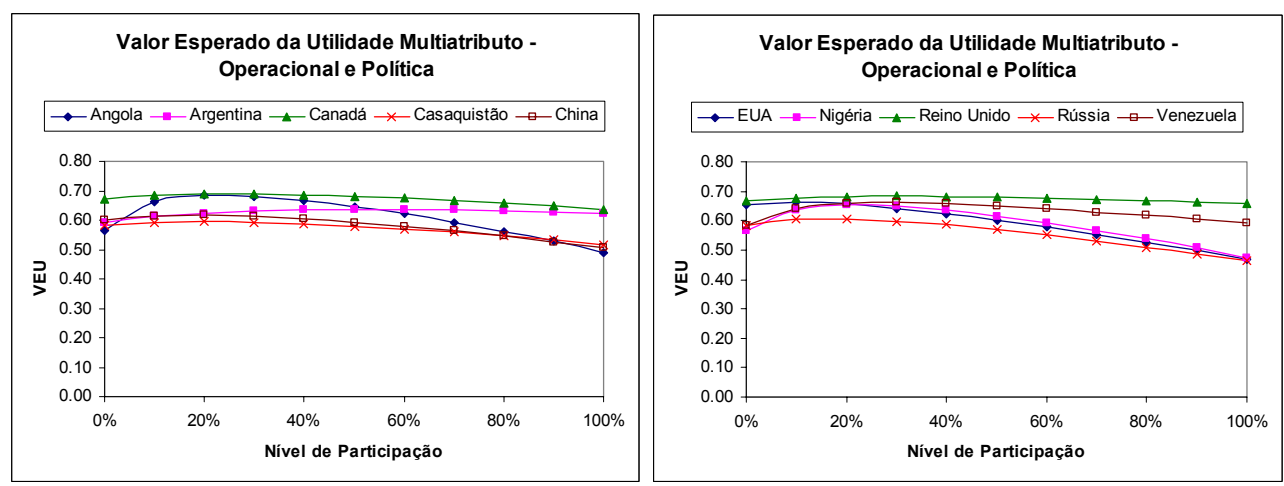

Figura 3 - Gráficos dos Valores Esperados das Utilidades operacional e política $\left(\operatorname{VEU}_{\text {op,pol, }}\right)$.

Tabela 6 - Classificação dos projetos nacionais considerando os riscos operacionais e políticos.

\begin{tabular}{|c|c|c|c|}
\hline Países & VEU $_{\text {op,pol }}$ & Participação ótima & Classificação \\
\hline Angola & 0,6854 & $20 \%$ & $2^{\circ}$ \\
\hline Argentina & 0,6365 & $50 \%$ & $7^{\circ}$ \\
\hline Canadá & 0,6886 & $30 \%$ & $1^{\circ}$ \\
\hline Casaquistão & 0,5942 & $20 \%$ & $10^{\circ}$ \\
\hline China & 0,6183 & $20 \%$ & $8^{\circ}$ \\
\hline EUA & 0,6641 & $10 \%$ & $4^{\circ}$ \\
\hline Nigéria & 0,6555 & $20 \%$ & $6^{\circ}$ \\
\hline Reino Unido & 0,6836 & $30 \%$ & $3^{\circ}$ \\
\hline Rússia & 0,6065 & $20 \%$ & $9^{\circ}$ \\
\hline Venezuela & 0,6612 & $30 \%$ & $5^{\circ}$ \\
\hline
\end{tabular}




\subsection{Risco tecnológico}

Tabela 7 - Utilidade do desenvolvimento tecnológico.

\begin{tabular}{|c|c|c|c|}
\hline Países & $\mathrm{U}_{\mathrm{ld}}$ & $\mathrm{U}_{\mathrm{pr}}$ & $\mathrm{U}_{\text {tec }}(\mathrm{ld}, \mathrm{pr})$ \\
\hline Angola & 0,99654 & 0,83245 & 0,91449 \\
\hline Argentina & 0,02110 & 0,40205 & 0,21157 \\
\hline Canadá & 0,65987 & 0,64636 & 0,65311 \\
\hline Casaquistão & 0,08808 & 0,19830 & 0,14319 \\
\hline China & 0,13737 & 0,59943 & 0,36840 \\
\hline EUA & 0,99971 & 0,99633 & 0,99802 \\
\hline Nigéria & 0,98802 & 0,42631 & 0,70717 \\
\hline Reino Unido & 0,84060 & 0,85852 & 0,84956 \\
\hline Rússia & 0,41647 & 0,55059 & 0,48353 \\
\hline Venezuela & 0,99872 & 0,99552 & 0,99712 \\
\hline
\end{tabular}

A introdução da variável tecnológica exige que os empresários, agora de posse de um conjunto maior de informações, explicitem novamente sua preferência. Trata-se de novo de um tradeoff, só que dessa vez mais complexo, pois não engloba apenas os fatores operacionais e políticos, mas também os tecnológicos.

$$
U_{o p, p o l, t e c}[V P L, R P,(l d-p r)]=k_{o p} \times U_{o p}(V P L)+k_{p o l} \times U_{p o l}(R P)+k_{t e c} \times U_{t e c}(l d, p r)
$$

No novo processo de entrevistas os tomadores de decisão mostraram-se indiferentes entre os projetos na Argentina, em Angola e na Nigéria.

$$
\begin{aligned}
U_{o p, p o l, t e c}\left[V P L_{A r g}, R P_{A r g},\left(l d_{A r g}, p r_{A r g}\right)\right] & =U_{o p, p o l, t e c}\left[V P L_{A n g}, R P_{A n g},\left(l d_{A n g}, p r_{A n g}\right)\right]= \\
& =U_{o p, p o l, t e c}\left[V P L_{N i g}, R P_{N i g},\left(l d_{N i g}, p r_{N i g}\right)\right] \\
U_{o p, p o l, t e c}[280 ; 62,20 ;(100 m, 3000 m)] & =U_{o p, p o l, t e c}[1000 ; 91,56 ;(2000 m, 4000 m)]= \\
& =U_{o p, p o l, t e c}[900 ; 87,11 ; 1(750 m, 3050 m)]
\end{aligned}
$$

Temos um sistema com três equações e três incógnitas:

$$
\begin{aligned}
& \mid\left[k_{\text {op }} \times U_{o p}(280)\right]+\left[k_{\text {pol }} \times U_{\text {pol }}(62,20)\right]+\left[k_{\text {tec }} \times U_{\text {tec }}(100 m, 3000 m)\right]= \\
& =\left[k_{\text {op }} \times U_{\text {op }}(1000)\right]+\left[k_{\text {pol }} \times U_{\text {pol }}(91,56)\right]+\left[k_{\text {tec }} \times U_{\text {tec }}(2000 m, 4000 m)\right] \\
& {\left[k_{o p} \times U_{o p}(1000)\right]+\left[k_{p o l} \times U_{p o l}(91,56)\right]+\left[k_{\text {tec }} \times U_{\text {tec }}(2000 m, 4000 m)\right]=} \\
& =\left[k_{o p} \times U_{o p}(900)\right]+\left[k_{p o l} \times U_{p o l}(87,11)\right]+\left[k_{\text {tec }} \times U_{\text {tec }}(1750 \mathrm{~m}, 3050 \mathrm{~m})\right] \\
& k_{o p}+k_{p o l}+k_{\text {tec }}=1 \\
& \begin{array}{l}
k_{o p}=0,86742 \\
k_{\text {pol }}=0,11478 \\
k_{\text {tec }}=0,01780
\end{array}
\end{aligned}
$$

$\mathrm{O} \mathrm{VEU}_{\mathrm{op}, \mathrm{pol}, \text { tec }}$ de cada um dos projetos nacionais, levando em consideração todos os riscos operacionais, políticos e tecnológicos, com suas importâncias relativas, é obtido pela equação (16): 


$$
\begin{aligned}
& V E U_{o p, p o l, t e c}=k_{o p}\left\{p \times\left[\frac{e^{-c(-100)}-e^{-c(V P L)}}{e^{-c(-100)}-e^{-c(1000)}}\right]+(1-p) \times\left[\frac{e^{-c(-100)}-e^{-c(C)}}{e^{-c(-100)}-e^{-c(1000)}}\right]\right\}+ \\
& +k_{p o l} \times\left[\frac{e^{-2 c(91,56)}-e^{-2 c(R P)}}{e^{-2 c(91,56)}-e^{-2 c(23,84)}}\right]+k_{t e c} \times\left[\frac{\frac{1}{\left(1+76,5 e^{-0,005(l d)}\right)}+\frac{1}{\left(1+600 e^{-0,002(p r)}\right)}}{2}\right]
\end{aligned}
$$

Após a definição da função-utilidade multiatributo, cabe ao analista identificar a consistência dos valores encontrados para a importância relativa de cada um dos atributos. Essa etapa procura verificar se a formulação está realmente reproduzindo as preferências dos tomadores de decisão. Caso contrário, todo o processo deve ser revisado e a função-utilidade reformulada.

Os dois gráficos a seguir demonstram como houve a otimização do valor esperado, que conjuga a utilidade operacional, política e tecnológica dos tomadores de decisão para cada um dos projetos analisados.
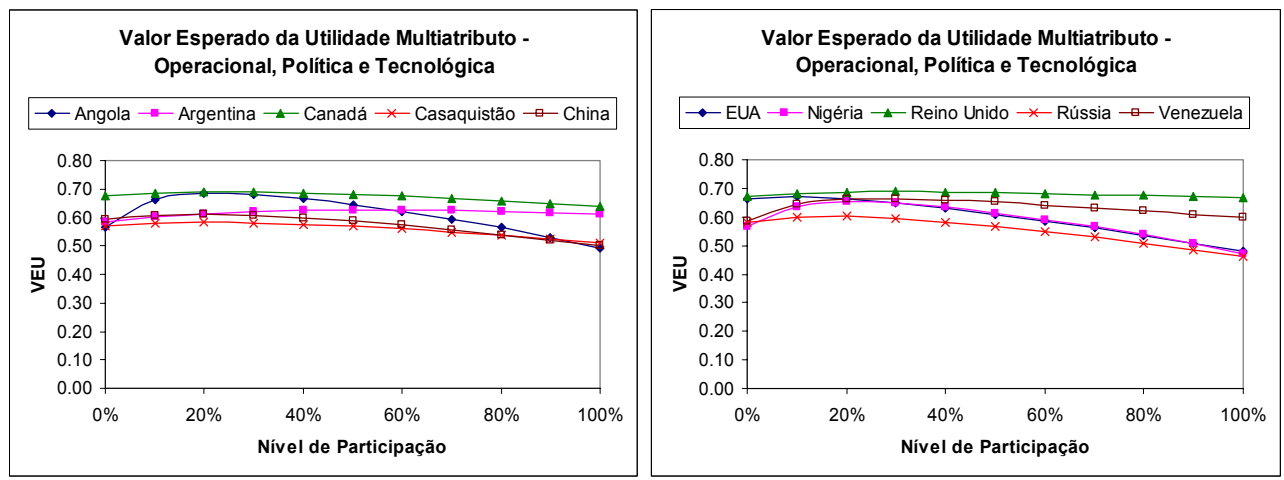

Figura 4 - Gráficos dos Valores Esperados das Utilidades operacional, política e tecnológica $\left(\mathrm{VEU}_{\mathrm{op}, \mathrm{pol}, \mathrm{tec}}\right)$

Tabela 8 - Classificação dos projetos nacionais considerando os riscos operacionais, políticos e tecnológicos.

\begin{tabular}{|c|c|c|c|}
\hline Países & VEU $_{\text {op,pol,tec }}$ & Participação ótima & Classificação \\
\hline Angola & 0,6846 & $20 \%$ & $3^{\circ}$ \\
\hline Argentina & 0,6264 & $50 \%$ & $7^{\circ}$ \\
\hline Canadá & 0,6902 & $30 \%$ & $1^{\circ}$ \\
\hline Casaquistão & 0,5832 & $20 \%$ & $10^{\circ}$ \\
\hline China & 0,6119 & $20 \%$ & $8^{\circ}$ \\
\hline EUA & 0,6712 & $10 \%$ & $4^{\circ}$ \\
\hline Nigéria & 0,6520 & $20 \%$ & $6^{\circ}$ \\
\hline Reino Unido & 0,6885 & $30 \%$ & $2^{\circ}$ \\
\hline Rússia & 0,6014 & $20 \%$ & $9^{\circ}$ \\
\hline Venezuela & 0,6638 & $30 \%$ & $5^{\circ}$ \\
\hline
\end{tabular}


Tabela 9 - Evolução da classificação dos projetos nacionais.

\begin{tabular}{|c|c|c|c|}
\hline Países & $\begin{array}{c}\text { Classificação com } \\
\text { Preferências Operacionais }\end{array}$ & $\begin{array}{c}\text { Classificação com } \\
\text { Preferências Operacionais } \\
\text { e Políticas }\end{array}$ & $\begin{array}{c}\text { Classificação com } \\
\text { Preferências Operacionais, } \\
\text { Políticas e Tecnológicas }\end{array}$ \\
\hline Angola & $1^{\circ}$ & $2^{\circ}$ & $3^{\circ}$ \\
\hline Argentina & $4^{\circ}$ & $7^{\circ}$ & $7^{\circ}$ \\
\hline Canadá & $7^{\circ}$ & $10^{\circ}$ & $10^{\circ}$ \\
\hline Casaquistão & $9^{\circ}$ & $8^{\circ}$ & $8^{\circ}$ \\
\hline China & $6^{\circ}$ & $4^{\circ}$ & $4^{\circ}$ \\
\hline EUA & $1^{\circ}$ & $6^{\circ}$ & $2^{\circ}$ \\
\hline Nigéria & $2^{\circ}$ & $3^{\circ}$ & $9^{\circ}$ \\
\hline Reino Unido & $8^{\circ}$ & $9^{\circ}$ & $5^{\circ}$ \\
\hline Rússia & $5^{\circ}$ & $5^{\circ}$ & \\
\hline Venezuela & $3^{\circ}$ & & \\
\hline
\end{tabular}

Observamos que as classificações de alguns dos projetos sofreram bastantes alterações, principalmente daqueles localizados nas nações que apresentam maior estabilidade política, econômica e social, representando reduzido risco político, como o caso dos projetos canadense, britânico e norte-americano.

Cabe ressaltar que a priorização dos projetos com baixo risco político foi decisão exclusiva dos executivos da empresa fíctícia, que deram considerável importância à variável política frente à operacional e tecnológica, ou seja, pouco mais de $11 \%$.

Temos plena convicção de que a utilização da modelagem apresentada por executivos reais da indústria do petróleo em verdadeiras oportunidades exploratórias contribui muito para um enriquecimento de seus processos decisórios, permitindo-os hierarquizar a carteira de projetos reais de acordo com suas preferências. No entanto, como nosso estudo de caso apresenta informações estratégicas, de mercado, operacionais, tecnológicas e financeiras de uma empresa virtual, com oportunidades exploratórias e tomadores de decisão que também são fictícios, precisamos estar bastante conscientes de que, a partir desses dados fictícios, mas próximos da realidade atual, não são possíveis decisões reais a respeito dos melhores países para se explorar petróleo na atualidade, até porque isto muito depende do comportamento de cada investidor.

\section{Conclusões}

Apresentamos a Teoria da Utilidade Multiatributo com três atributos que jamais haviam sido integrados em um único modelo. Incorporamos a função-utilidade tecnológica desenvolvida a partir de Suslick et al. (2001) no modelo multiatributo de Nepomuceno (1997), que apresentava apenas as variáveis operacionais e políticas.

Chegamos à conclusão de que a priorização de investimentos em um setor como o de petróleo requer a participação de um grupo bastante multidisciplinar para a consideração de todos os aspectos que podem influenciar no futuro, positiva ou negativamente, a geração de fluxo de caixa de um projeto exploratório internacional. 
A seleção da MAUT como arcabouço teórico da modelagem de investimentos internacionais em E\&P de petróleo deveu-se ao fato de ela ser recomendada para a solução de problemas que envolvam objetivos conflitantes. Os investidores do setor de Óleo \& Gás valorizam a MAUT como metodologia quantitativa de apoio aos seus processos de tomada de decisão pois na maior parte dos casos eles se deparam em situações nas quais precisam realizar "trade-offs". Isto é, necessitam abrir mão de excelentes condições operacionais para reduzir o risco político do investimento - por exemplo, quando priorizam um prospecto britânico em relação a um nigeriano -, ou abrir mão da segurança política nacional para terem acesso a grandes possibilidades de avanços tecnológicos em produção em águas profundas - por exemplo, no caso em que optam por um prospecto venezuelano em detrimento de um canadense.

Uma grande vantagem da aplicação da ferramenta quantitativa apresentada nesse trabalho é que uma decisão de priorização de um projeto em relação a outros não é apresentada de forma intuitiva em uma "caixa preta", mas se encontra amparada em uma modelagem matemática robusta e transparente que apresenta um encadeamento lógico e racional de premissas e preferências por parte do decisor a respeito de cada um dos atributos considerados durante o processo.

Todas as funções-utilidade apresentadas nessa dissertação foram obtidas por especialistas que as consideram aquelas que melhor representam o comportamento da maioria das empresas de petróleo em seus processos decisórios. É fundamental que tenhamos clara a idéia de que não existem funções-utilidade certas ou erradas, mas, sim, empresas mais ou menos dispostas a enfrentar os riscos associados a cada uma das variáveis incertas modeladas.

Portanto, verificamos que a Teoria da Utilidade Multiatributo se aplica às condições reais e complexas do mercado petrolífero, onde projetos com suas vantagens e desvantagens para o tomador de decisão têm que ser selecionados de forma coerente, racional e menos instintiva, de modo que os escolhidos proporcionem uma otimização dos objetivos globais da empresa.

\section{Agradecimentos}

Agradecemos ao apoio da Financiadora de Estudos e Projetos (FINEP) e da Agência Nacional do Petróleo (ANP). Agradecemos também as valiosas sugestões dos revisores deste artigo que em muito contribuíram para aperfeiçoar nosso manuscrito. Nós, autores, assumimos plenamente a responsabilidade pelo conteúdo deste artigo.

\section{Referências Bibliográficas}

(1) Cozzolino, J.M. (1977). Management of oil and gas exploration risk. West Berlin, Cozzolino Associates.

(2) Folha de São Paulo (2002). Corrupção piora na América Latina, diz ONG. 29/08/2002.

(3) Global Invest (2003). Rankings soberanos das principais agências de classificação de risco. Relatório Mensal de Janeiro de 2003. Material consultado na Internet: $<$ http://www.globalinvest.com.br/relatorios/Cenar\%20jan-03.pdf>

(4) Gomes, L.F.A.M.; Gomes, C.F.S. \& De Almeida, A.T. (2002). Tomada de Decisão Gerencial: Enfoque Multicritério. $1^{\mathrm{a}}$ ed., Ed. Atlas S.A, São Paulo. 
(5) Hargreaves, D.; Eden-Green, M. \& Devaney, J. (1994). World index of resources and population. England, Dartmouth.

(6) Margueron, M.V. (2003). Processo de Tomada de Decisão sob Incerteza em Investimentos Internacionais na Exploração \& Produção de Petróleo "Offshore": Uma Abordagem Multicritério. Tese de M.Sc., COPPE/Universidade Federal do Rio de Janeiro (UFRJ), Rio de Janeiro, RJ.

(7) Margueron, M.V. \& Carpio, L.G.T. (2003). Processo de Tomada de Decisão em Projetos Internacionais de Risco na Exploração \& Produção "Offshore" de Petróleo: Um Enfoque Multicritério. Anais do XXXV Simpósio Brasileiro de Pesquisa Operacional, 1821-1832.

(8) Nepomuceno, F. (1997). Tomada de Decisão em Projetos de Risco na Exploração de Petróleo. Tese de D.Sc., Universidade Estadual de Campinas, Campinas, SP.

(9) Newendorp, P.D. (1975). Decision Analysis for Petroleum Exploration. $1^{\text {a }}$ ed., PPC Books, Oklahoma.

(10) Suslick, S.B.; Furtado, R. \& Nepomuceno, F. (2001). Integrating Technological and Financial Uncertainty for Offshore Oil Exploration: An Application of Multiobjetive Decision Analysis. Report SPE 68579, Society of Petroleum Engineers (SPE).

(11) The World Factbook (2002). Publicação da CIA. Material consultado na Internet: $<$ http://www.odci.gov/cia/publications/factbook $>$

(12) Walls, M.R. (1995). Corporate risk tolerance and capital allocation: a practical approach to implementing an exploration risk policy. Jorn. of Petroleum Technology, 47(4), 307-311.

(13) Walls, M.R. \& Dyer, J.S. (1996). Risk propensity and firm performance: A study of the petroleum exploration industry. Management Science, 42(7), 1004-1021.

\section{Anexo 1}

Demonstração matemática da equação (6).

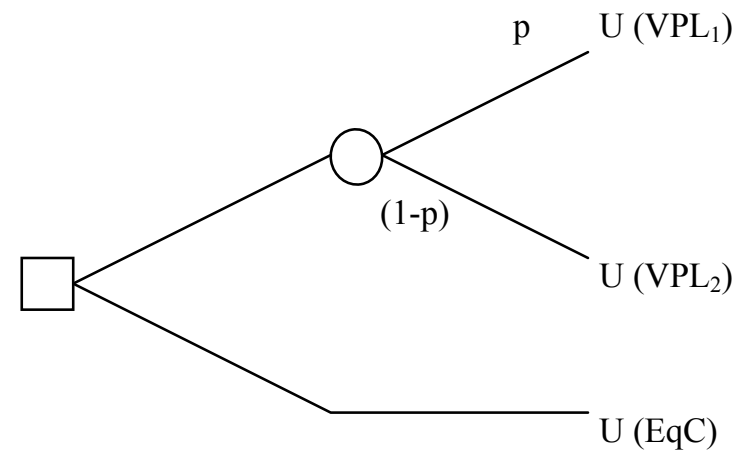

Figura 5 - Árvore de decisão para se definir o Valor Esperado da Utilidade. 


$$
V E U=\left(p \times U\left(V P L_{1}\right)+\left[(1-p) \times U\left(V P L_{2}\right)\right]\right.
$$

Aplicando a equação (5) à definição de que a utilidade do Equivalente Certo corresponde ao Valor Esperado da Utilidade (VEU), conforme mostra a formulação (3), temos o seguinte valor para o Equivalente Certo dos projetos no setor "upstream":

$$
\begin{aligned}
& U(E q C)=V E U \\
& -e^{-c(E q C)}=p\left(-e^{-c\left(V P L_{1}\right)}\right)+\left[(1-p)\left(-e^{-c\left(V P L_{2}\right)}\right)\right] \\
& e^{-c(E q C)}=p e^{-c\left(V P L_{1}\right)}+(1-p) e^{-c\left(V P L_{2}\right)} \\
& -c(E q C)=\ln \left(p e^{-c\left(V P L_{1}\right)}+(1-p) e^{-c\left(V P L_{2}\right)}\right) \\
& E q C=-\frac{1}{c} \ln \left(p e^{-c\left(V P L_{1}\right)}+(1-p) e^{-c\left(V P L_{2}\right)}\right)
\end{aligned}
$$

Ao considerarmos o nível ótimo de participação (np) em cada um dos projetos passamos a trabalhar com a equação (6).

$$
E q C(n p)=-\frac{1}{c} \ln \left(p e^{-c\left(V P L_{1}\right) n p}+(1-p) e^{-c\left(V P L_{2}\right) n p}\right)
$$

\section{Anexo 2}

Demonstração matemática da função-utilidade do Equivalente Certo explicitada pela equação (10).

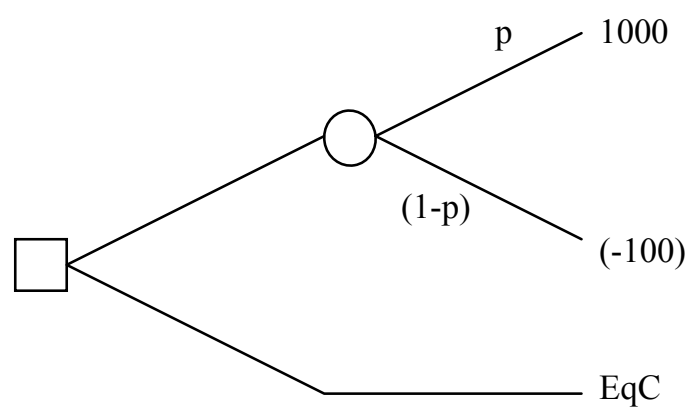

Figura 6 - Árvore de decisão para se definir a função-utilidade do Equivalente Certo.

A Figura 6 ilustra o jogo de referência dentro do espaço conseqüência definido para a variável operacional, ou seja, o VPL mais positivo - US\$ 1000 milhões - e o Custo mais negativo - (-US\$ 100 milhões).

Considerando as equações (3) e (5) e devido a que todas as utilidades apresentadas nesse trabalho foram normalizadas em uma escala de 0 a 1, obtemos a utilidade do Equivalente Certo: 


$$
\begin{aligned}
& E q C=(p \times 1000)+[(1-p) \times(-100)] \\
& U(E q C)=[p \times U(1000)]+[(1-p) \times U(-100)] \\
& \left(-e^{-c(E q C)}\right)=\left[p \times\left(-e^{-c(1000)}\right)\right]+\left(-e^{-c(-100)}\right)-\left[p \times\left(-e^{-c(-100)}\right)\right] \\
& \left(-e^{-c(E q C)}\right)-\left(-e^{-c(-100)}\right)=p \times\left[\left(-e^{-c(1000)}\right)-\left(-e^{-c(-100)}\right)\right] \\
& V E U_{o p}=U(E q C)=\frac{e^{-c(-100)}-e^{-c(E q C)}}{e^{-c(-100)}-e^{-c(1000)}}
\end{aligned}
$$

\section{Anexo 3}

Demonstração matemática da função-utilidade do Risco Político explicitada pela equação (12).

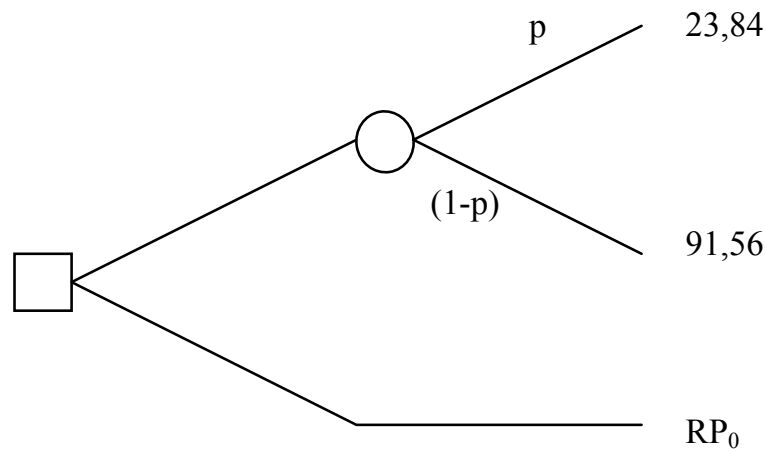

Figura 7 - Árvore de decisão para se definir a função-utilidade política normalizada.

A Figura 7 ilustra o jogo de referência apresentado a um típico tomador de decisão em negócios internacionais da indústria do petróleo, a fim de obter as curvas de preferência frente a uma real oportunidade de investimento.

Essa loteria representa o projeto preferível do ponto de vista político - o canadense -, com probabilidade de sucesso p, e o menos preferível e de maior risco político - o angolano -, com probabilidade (1-p).

Considerando a equação (5), obtemos a utilidade do risco político:

$$
\begin{aligned}
& R P_{0}=(p \times 23,84)+[(1-p) \times(91,56)] \\
& U\left(R P_{0}\right)=[p \times U(23,84)]+[(1-p) \times U(91,56)] \\
& \left(-e^{-2 c\left(R P_{0}\right)}\right)=\left[p \times\left(-e^{-2 c(23,84)}\right)\right]+\left(-e^{-2 c(91,56)}\right)-\left[p \times\left(-e^{-2 c(91,56)}\right)\right] \\
& \left(-e^{-2 c\left(R P_{0}\right)}\right)-\left(-e^{-2 c(91,56)}\right)=p \times\left[\left(-e^{-2 c(23,84)}\right)-\left(-e^{-2 c(91,56)}\right)\right] \\
& U\left(R P_{0}\right)=\frac{e^{-2 c(91,56)}-e^{-2 c\left(R P_{0}\right)}}{e^{-2 c(91,56)}-e^{-2 c(23,84)}}
\end{aligned}
$$

